\title{
Pharmacist-managed Diabetes Clinic in Malaysia - Does the Number of Follow-up Visits Really Matter?
}

\author{
Mithali Jacqueline Abdullah', Mei Mei Tew ${ }^{2 *}$, Pei Hoon Tan ${ }^{3}$, Jing Hui Koh', Nurhayati Md Osman ${ }^{3}$, \\ Huan Keat Chan ${ }^{4}$ \\ ${ }^{1}$ Hospital Yan, Kedah, MALAYSIA. \\ ${ }^{2}$ Clinical Research Centre, Hospital Sultan Abdul Halim, Kedah, MALAYSIA. \\ ${ }^{3}$ Pharmacy Department, Hospital Sultan Abdul Halim, Kedah, MALAYSIA. \\ ${ }^{4}$ Clinical Research Centre, Hospital Sultanah Bahiyah, Kedah, MALAYSIA.
}

\section{Received: 17 February 2018; \\ Accepted: 16 May 2018 \\ *Correspondence to: \\ Tew Mei Mei,}

Clinical Research Centre, Hospital Sultan Abdul Halim, Jalan Lencongan Timur, Bandar Aman Jaya, 08000, Sungai Petani, Kedah, MALAYSIA. Email:tewmeimei@gmail.com

Copyright: (c) the author(s),publisher and licensee Indian Academy of Pharmacists. This is an open-access article distributed under the terms of the Creative Commons Attribution Non-Commercial License, which permits unrestricted non-commercial use, distribution, and reproduction in any medium, provided the original work is properly cited.

\begin{abstract}
Background: The involvement of pharmacists in diabetes education and management has been shown to improve patient outcomes worldwide. The pharmacist-managed Diabetes Medication Therapy Adherence Clinic (DMTAC) has been introduced across public healthcare settings in Malaysia to enhance patient adherence to treatment. Objective: This study was designed to assess the effectiveness of the DMTAC in optimizing the glycemic control of diabetic patients, and to subsequently identify the relationship between the number of follow-up visits and the glycemic control. Method: This was a retrospective cohort study performed in the department of Pharmacy, Sultan Abdul Halim Hospital, Kedah, Malaysia. All the patients, who had type 2 diabetes mellitus, made at least four visits to the pharmacist-managed DMTAC during May 2014 and April 2016, and had their HbA1c levels tested once each before enrolled in the DMTAC and within the same month of the last visit, were included. Percentage of patients achieving the targeted $\mathrm{HbA} 1 \mathrm{c}$ level of $7.5 \%$ or below, and factors associated with the achievement of the targeted $\mathrm{HbA} 1 \mathrm{c}$ level were recorded. Results: Only $21 \%$ of patients managed to achieve the targeted $\mathrm{HbA} 1 \mathrm{c}$ level. Higher baseline HbA1c (OR: 2.34; 95\% Cl: 1.14, 4.79) and FPG (OR: 1.41; 95\% Cl: 1.02, 1.95) levels were more likely to lead to a non-optimized $\mathrm{HbA} 1 \mathrm{c}$ level. Conclusion: Despite the effectiveness of the DMTAC in improving the glycemic control, majority of the patients did not achieve targeted level. Number of visits to the DMTAC is not a determinant of the targeted outcome and should not be used as discharging criteria in DMTAC.
\end{abstract}

Key words: Pharmacist-managed, Diabetes clinic, HbA1c, Visit, Malaysia.

\section{INTRODUCTION}

Diabetes mellitus (DM) has been imposing a significant burden on health care system due to its lifelong complications and high management costs. ${ }^{[1]}$ Over the past three decades, the global prevalence of DM had risen steadily from $4.3 \%$ to $9 \%$ in men, and from $5 \%$ to $7.9 \%$ in women. ${ }^{[2]}$ Equally of concern is the increasing number of type 2 diabetes mellitus (T2DM) patients in middleand low-income countries. ${ }^{[3]}$ For instance, in Malaysia, a population-based survey revealed that the prevalence of T2DM increased by more than twofold from $11.6 \%$ in 2006 to $22.9 \%$ in $2013 \cdot{ }^{[4]}$ As a consequence, the healthcare expenditure on DM has constituted approximately $16 \%$ of the total healthcare expenditure in Malaysia, equivalent to USD 1.01 million annually. ${ }_{[}^{[5]}$

Strict glycemic control, which is mainly achieved through adherence to treatment and good self-care behaviors, undeniably plays a pivotal role in DM management. ${ }^{[6]}$ Within this context, the involvement of pharmacists in diabetes education and management has been shown to improve patient outcomes worldwide. ${ }^{[7-10]}$ Since the past decade, the pharmacist-managed Diabetes Medication Therapy Adherence Clinic (DMTAC) has also been introduced across public healthcare settings in Malaysia, serving as an important strategy of the Ministry of Health, Malaysia, to enhance patient adherence to treatment, and thereby to reduce the incidence of DM-related complications..$^{[11,12]}$ Under this program, all patients referred to the DMTAC, who generally have poor glycemic control, are scheduled to meet a trained pharmacist monthly to receive education and counseling on diabetes and its complications; medications and the management of their side effects; selfmonitoring of blood glucose level; physical activity; and diet control. Besides, the pharmacotherapy plans of patients and their adherence to proper self-care are reviewed by pharmacists during each follow-up visit. ${ }^{[12]}$

Although the DMTAC was consistently shown to improve the medication adherence and glycemic control of diabetic patients, ${ }^{[11,12]}$ none of the existing studies had examined its effectiveness in helping patients to achieve the targeted $\mathrm{HbA} 1 \mathrm{c}$ level. In addition, notwithstanding the recommendation made by the Ministry of Health, Malaysia, to discharge patients from the DMTAC after at least eight visits, ${ }^{[13]}$ the impact of the number of follow-up visits on the glycemic control is still unknown. Hence, this study was designed to assess the effectiveness of the DMTAC in optimizing the glycemic control of diabetic patients, and to subsequently identify the relationship between the number of follow-up visits and the glycemic control.

\section{METHODS}

This was a retrospective cohort study undertaken at the Sultan Abdul Halim Hospital, a secondary care centre in the northern region of Malaysia. All the T2DM patients, who had at least four visits to the pharmacist-managed DMTAC during May 2014 and April 2016 and had their HbA1C levels tested once each before enrolled in the DMTAC and within the same month of 
the last visit, were included. Aside from that, the patients who had stage 3 to 5 chronic kidney disease (CKD) were excluded, as their HbA1c levels were likely to be affected by a wide range of CKD-related complications, such as the reduced life span of red blood cells and iron deficiency. ${ }^{[14]}$ All the information needed, including patient demographics (age, gender and ethnicity); number of visits to the DMTAC; comorbidities; medication history; $\mathrm{HbA1c}$ and fasting plasma glucose (FPG) levels; and the involvement in self-care practices (physical exercise, self-monitoring of blood glucose and dietary habits), were collected from the individual DMTAC clinical records and the e-Hospital Information System (e-HIS). The targeted HbA1c level for each patient was set at $7.5 \%$ as recommended by the Ministry of Health, Malaysia. ${ }^{[13]}$ Statistical Analysis: The data analysis was performed by using the Statistical Packages for Social Sciences (SPSS) version 21.0 (IBM, New York). Categorical variables were presented as frequencies and percentages, and numerical variables as means and standard deviations (SDs). The associations between two variables were tested using the Pearson's chi-square, Fisher's exact, independent t- or Mann-Whitney tests, as appropriate. On the other hand, the differences between the baseline and post-DMTAC HbA1c and FPG levels were tested using the paired t-tests. The factors potentially influencing the control of $\mathrm{HbA1c}$ level, including the number of visits to the MTAC, were tested using the backward stepwise multiple logistic regression analysis $(\mathrm{p} \leq 0.3$ in simple logistic regression analysis used as the cut off for variable selection), in which the results were expressed as odds ratios (ORs) and 95\% confidence intervals (CIs). Ethics approval: The study was registered with the National Medical Research Register (NMRR) with registration number NMRR-16-488-30164 and was approved by the Malaysian National Medical Research Ethical Committee (MREC) (approval reference: (5)/KKM/NIHSEC/P16-585).

\section{RESULTS}

A total of 53 patients ( 28 male and 25 female) with a median age of 57 years were included in the study. On average, they had made 9 visits to the DMTAC. The vast majority of them were found to have hypertension $(90.6 \%)$ and hyperlipidemia (64.2\%). Most of them were also reported to be insulin users $(94.3 \%)$, while only approximately half of them practiced strict diet control. Furthermore, $86.8 \%$ of them did not perform regular physical exercise. On top of that, interestingly, as compared with the patients with at least 8 visits to the DMTAC, those who had a lower number of visits were found to have higher adherence to diet control (35.7\% versus $80 \%$ ) (Table 1$)$.

Overall, irrespective of the number of visits, lifestyle and drug-taking behaviors, the HbA1c and FPG of the patients were found to reduce by $1.3 \%(\mathrm{p}<0.001)$ and $2.8 \mathrm{mmol} / \mathrm{L}(\mathrm{p}<0.001)$, respectively (Table 2$)$. However, only $21 \%$ of them managed to achieve the targeted HbA1c level. Of all the patients who failed to achieve the targeted $\mathrm{HbA1c}$ level, more than half had made at least 8 visits to the DMTAC (Figure 1).

\begin{tabular}{|c|c|c|c|c|c|c|c|}
\hline \multirow{2}{*}{ Variable } & \multicolumn{2}{|l|}{ Total } & \multicolumn{2}{|l|}{$<=8$ visits } & \multicolumn{2}{|l|}{$>8$ visits } & \multirow{2}{*}{$p$ value } \\
\hline & n (\%) & mean $(\mathrm{sd})$ & n (\%) & mean (sd) & n (\%) & mean (sd) & \\
\hline Age (years) & & $57.00(11.00)^{*}$ & & $57.12(6.62)$ & & $53.46(10.28)$ & $0.135^{\dagger}$ \\
\hline \multicolumn{8}{|l|}{ Gender } \\
\hline Male & $28(52.8)$ & & $14(56.0)$ & & $14(50.0)$ & & $0.785^{\ddagger}$ \\
\hline Female & $25(47.2)$ & & $11(44.0)$ & & $14(50.0)$ & & \\
\hline \multicolumn{8}{|l|}{ Ethnicity } \\
\hline Malay & $36(67.9)$ & & $17(68.0)$ & & $19(67.9)$ & & $0.441^{\ddagger}$ \\
\hline Chinese & $4(7.5)$ & & $3(12.0)$ & & $1(3.6)$ & & \\
\hline Indian & $13(24.5)$ & & $5(20.0)$ & & $8(28.6)$ & & \\
\hline Body weight (kg) & & $71.6(12.00)$ & $70.93(10.00)$ & & $72.08(13.29)$ & & $0.737^{\dagger}$ \\
\hline No. of DMTAC visits & & $9.00(5.00)^{*}$ & & $6.00(2.00)^{\star}$ & & $11.00(2.00)^{\star}$ & $<0.001^{\S}$ \\
\hline$I H D$ & $21(39.6)$ & & $10(40.0)$ & & $11(39.3)$ & & $0.958^{\ddagger}$ \\
\hline Hypertension & 48 (90.6) & & $22(88.0)$ & & $26(92.9)$ & & $0.658^{\ddagger}$ \\
\hline Hyperlipidemia & $34(64.2)$ & & $10(40.0)$ & & $24(85.7)$ & & $0.001^{\ddagger}$ \\
\hline No. of Medications & & $7.96(2.32)$ & & $7.36(2.00)$ & & $8.50(2.49)$ & $0.074^{+}$ \\
\hline Insulin & $50(94.3)$ & & $22(88.0)$ & & $28(100.0)$ & & $0.098 \|$ \\
\hline$O A D$ & $44(83.0)$ & & $21(84.0)$ & & $23(82.1)$ & & $0.857^{\ddagger}$ \\
\hline Physical exercise & $7(13.2)$ & & $2(8.0)$ & & $5(17.9)$ & & $0.290^{\ddagger}$ \\
\hline SMBG & $53(100.0)$ & & $25(100.0)$ & & $28(100.0)$ & & - \\
\hline Diet control & $30(56.6)$ & & $20(80.0)$ & & $10(35.7)$ & & $0.001^{\ddagger}$ \\
\hline Baseline HBA1c level & & $10.63(1.68)$ & & $10.11(1.62)$ & & $10.98(1.66)$ & 0.066 \\
\hline Baseline FBS level & & $11.11(3.27)$ & & $10.80(3.74)$ & & $11.31(2.97)$ & 0.585 \\
\hline
\end{tabular}

DMTAC=Diabetic Medication Therapy Adherence Clinic; FBS=fasting blood sugar, IHD = ischemic heart disease, OAD= oral antidiabetic drug, $\mathrm{SMBG}=$ self-monitoring blood glucose, sd= standard deviation.

*Presented as median (interquartile range); 'Independent-test; †Pearson's chi-square test; §Mann-Whitney test; " Fisher's exact test. 


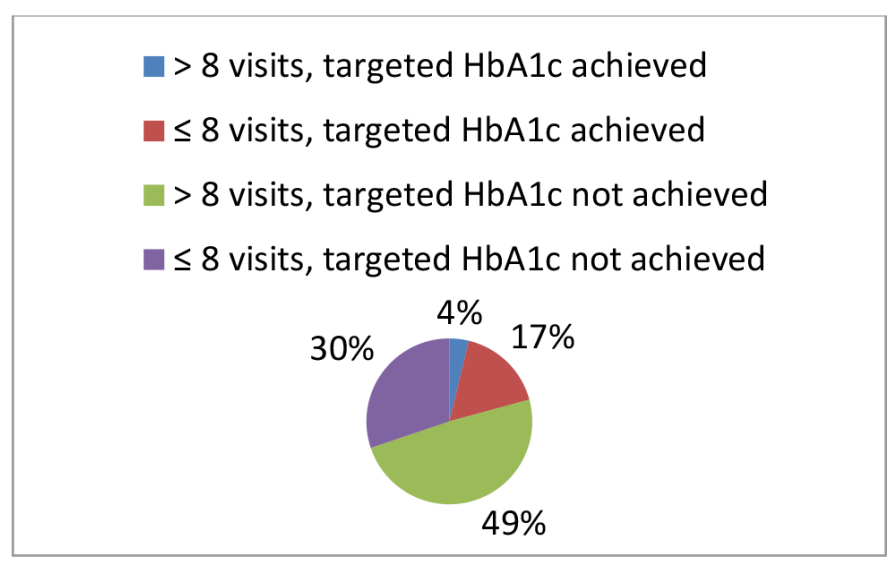

Figure 1: Distribution of the patients achieving the targeted $\mathrm{HbA} 1 \mathrm{C}$ level by the number of their visits to the DMTAC.

\begin{tabular}{|c|c|c|c|c|c|c|}
\hline & Pre & Post & Mean diff & $95 \%$ & & $\begin{array}{l}p \\
\text { value* }\end{array}$ \\
\hline & & & & & & \\
\hline HbA1c & $10.63(1.68)$ & $9.32(1.71)$ & -1.32 & $(-1.82$ & $,-0.81)$ & $<0.001$ \\
\hline FBS & $11.11(3.27)$ & $8.30(3.70)$ & -2.81 & $(-3.92$ & $,-1.71)$ & $<0.001$ \\
\hline
\end{tabular}

$\mathrm{Cl}=$ confidence interval, $\mathrm{d}=$ standard deviation, $\mathrm{FBS}=$ fasting blood glucose . ${ }^{*}$ Paired t-test

The results of both the simple and multiple logistic regression analyses showed that the number of visits was not significantly associated with the achievement of the targeted HbA1c level. Nevertheless, higher baseline HbA1c (OR: 2.34; 95\% CI: 1.14, 4.79) and FPG (OR: 1.41; 95\% CI: 1.02, $1.95)$ levels were confirmed to be more likely to lead to a non-optimized HbA1c level (Table 3 and 4).

\section{DISCUSSION}

To the best knowledge of the investigators, this is the first study on the impact of the pharmacist-managed DMTAC on the achievement of the targeted HbA1c level in Malaysia. It is also the first attempt to establish the relationship between the number of visits to the DMTAC and the glycemic control of patients. The findings could be used to help in the revision of the current protocol of the DMTAC, which recommends that patients could be discharged from the clinic after eight follow-up visits.

A significant reduction in both the HbA1c and FPG levels was observed in the patients, who had made at least four visits to the DMTAC, in this study. The magnitude of reduction in HbA1c is similar to that of a previous study, which demonstrated that the DMTAC managed to reduce the HbA1c level of T2DM patients from $9.66 \%$ to $8.47 \%{ }^{[15]}$ Such findings have been inspiring, as a number of the similar studies on pharmacist-managed diabetes programs have demonstrated a lower HbA1c reduction, in the range of 0.4 to $0.5 \%$, over a longer study period. ${ }^{[16]}$ However, notwithstanding the positive results, a longer follow-up period is still needed to confirm the sustainability of the benefits of the MTAC.

Intriguingly, it is also found that all the patients practiced SMBG following their participation in the DMTAC. Anyhow, their poor commitment to the other aspects of self-care, particularly strict diet control and physical

\begin{tabular}{|c|c|c|c|c|}
\hline \multirow{2}{*}{$\begin{array}{l}\text { Variables } \\
\text { Age (years) }\end{array}$} & \multirow{2}{*}{$\begin{array}{l}\text { Crude OR } \\
0.99\end{array}$} & \multicolumn{2}{|c|}{$95 \% \mathrm{Cl}$} & \multirow{2}{*}{$\begin{array}{l}\text { p value } \\
0.849\end{array}$} \\
\hline & & $(0.92$ & ;1.91) & \\
\hline \multicolumn{5}{|l|}{ Gender } \\
\hline Male & 1 & & & \\
\hline Female & 1.75 & $(0.45$ & ;6.88) & 0.423 \\
\hline \multicolumn{5}{|l|}{ Ethnicity } \\
\hline Malay & 1 & & & \\
\hline Chinese & 1.0 & $(0.09$ & ;10.87) & $>0.95$ \\
\hline Indian & 4.0 & $(0.45$ & ; 35.21) & 0.212 \\
\hline Body Weight & 1.02 & $(0.96$ & ;1.08) & 0.602 \\
\hline No of visits & 1.10 & $(0.91$ & ;1.32) & 0.324 \\
\hline \multicolumn{5}{|l|}{$\mathrm{IHD}$} \\
\hline No & 1 & & & \\
\hline Yes & 0.463 & $(0.12$ & ;1.78) & 0.261 \\
\hline \multicolumn{5}{|l|}{ Hypertension } \\
\hline No & 1 & & & \\
\hline Yes & 1.053 & $(0.11$ & ; 10.49) & 0.965 \\
\hline \multicolumn{5}{|l|}{ Hyperlipidemia } \\
\hline No & 1 & & & \\
\hline Yes & 0.374 & $(0.10$ & ;1.45) & 0.154 \\
\hline Number of medications & 1.16 & $(0.89$ & ;1.57) & 0.336 \\
\hline Insulin* & - & - & - & - \\
\hline \multicolumn{5}{|l|}{ OAD } \\
\hline Yes & 1 & & & \\
\hline No & 1.11 & $(0.20$ & ;6.30) & 0.905 \\
\hline \multicolumn{5}{|l|}{ Physical exercise } \\
\hline Yes & 1 & & & \\
\hline No & 0.6 & $(0.07$ & ; 5.58) & 0.653 \\
\hline $\mathrm{SMBG}^{+}$ & - & - & - & - \\
\hline \multicolumn{5}{|l|}{ Diet Control } \\
\hline Yes & 1 & & & \\
\hline No & 4.5 & $(0.87$ & ;23.37) & 0.074 \\
\hline Baseline HbA1c level & 2.49 & $(1.31$ & ;4.72) & 0.005 \\
\hline Baseline FBS level & 1.47 & $(1.10$ & ;1.96) & 0.009 \\
\hline
\end{tabular}

$\mathrm{Cl}=$ confidence Interval, $\mathrm{IHD}=$ ischemic heart disease, $\mathrm{OAD}=$ oral antidiabetic drug, $\mathrm{OR}=$ odds ratio, SMBG = self-monitoring blood glucose, sd= standard deviation

*the number of non-insulin users is too small for simple logistic regression analysis

tall the patients were reported to practice SMBG.

exercise, is still of concern, even though such practices were not shown to significantly affect the achievement of the targeted $\mathrm{HbA1c}$ level in this study. Generally, compared with a change in the pharmacotherapy plan, self-care behaviours would require a longer study period to demonstrate their impacts, especially when the achievement of a specific HbA1c level was used as the endpoint. Nonetheless, despite the negative findings, the roles of all these self-care practices in long-term disease management and reducing diabetes-related complications have been well-established. ${ }^{[17-20]}$ Therefore, a strategy to overcome the widely reported barriers to self-care in T2DM patients, including their poor attitude and the lack of financial support and facilities, ${ }^{[11,22]}$ is necessary.

Moreover, irrespective of the improvement in the overall glycemic control and the practice of SMBG, it is noteworthy that the majority of the patients, 


\begin{tabular}{|c|c|c|c|}
\hline Variables & Adjusted OR* & $95 \% \mathrm{Cl}$ & $p$ value \\
\hline Baseline HbA1c level & 2.34 & $(1.14 ; 4.79)$ & 0.020 \\
\hline Baseline FBS level & 1.41 & $(1.02: 1.95)$ & 0.036 \\
\hline
\end{tabular}

*Backward stepwise multiple logistic regression analysis was applied. Multicollinearity and interaction term were checked and not found. Hosmer-Lemeshow test, $(p=0.288)$, classification table (overall correctly classified percentage $=79.2 \%$ ) and area under the ROC curve $(86.1 \%)$ were applied to check the model fitness.

including those who had made more than eight visits to the DMTAC, failed to achieve the targeted $\mathrm{HbA} 1 \mathrm{c}$ level of $7.5 \%$ as recommended by guidelines. ${ }^{[23,24]}$ In addition, conversely, the patients who had a lower number of visits were found to have a better diet control. In fact, on average, the patients in this study had completed nine follow-up visits. The findings indicate that the pharmacists had been unconfident to discharge most of the patients following eight visits, given the suboptimal glycemic control. As the number of visits to the DMTAC has been shown not to be significantly associated with the achievement of the targeted HbA1c level, there is a clear need to revise the existing protocol, which suggests the use of the number of visits as the main criterion for discharging patients. Instead, considering that the baseline HbA1c and FPG levels are the only two factors significantly affecting the targeted patient outcome, they should be used to guide the pharmacists in designing individualized pharmaceutical care plans and deciding the number of visits required by each patient.

Yet, this study has several limitations. First, its single-centre design and relatively small sample size has limited the generalizability of the findings. Hence, in order to recommend an actual policy change, future studies should involve more healthcare centres which operate the DMTAC and have a larger sample size. Besides, the limitation regarding the internal validity should be considered when interpreting the results on the benefits of the DMTAC, as there was no control group in this study. Nonetheless, the roles of pharmacists in helping diabetes patients through education and counselling have been widely explored and confirmed by the existing studies. Thus, this study aimed at putting the study on the DMTAC in a different context, focusing primarily on the relationship between the number of visits and the patient outcomes.

\section{CONCLUSION}

In conclusion, despite the effectiveness of the DMTAC in improving the glycemic control and self-care practices, the majority of the T2DM patients enrolled in the program, including those who had completed eight follow-up visits, did not achieve the HbA1c level of 7.5\%. The finding suggests that the number of visits to the DMTAC is not a determinant of the targeted outcome, and thus should not be used as the main criteria for discharging patients. On the other hand, the baseline HbA1c and FPG levels are shown to be associated with the achievement of the targeted $\mathrm{HbA1c}$ level, and therefore could be used to guide pharmacists in individualizing pharmaceutical care plans for each patient. However, further studies, particularly those with a larger sample size and a control group, are warranted to verify these findings.

\section{ACKNOWLEDGEMENT}

The authors would like to thank the Director General of Health for permission to publish this article.

\section{ABBREVIATIONS}

CKD (Chronic Kidney Disease); DMTAC (Diabetes Medication Therapy Adherence Clinic); FBG (Fasting Blood Glucose); HbA1c (Glycated haemoglobin); MTAC (Medication Therapy; Adherence Clinic); T2DM (Type 2 Diabetes Mellitus)

\section{FUNDING INFORMATION: NIL}

\section{CONFLICTS OF INTERESTS: NIL}

\section{REFERENCES}

1. Kakkar R. Rising burden of diabetes-public health challenges and way out. Nepal J Epidemiol. 2016;6(2):557-9.

2. NCD Risk Factor Collaboration. Worldwide trends in diabetes since 1980: A pooled analysis of 751 population-based studies with 4.4 million participants. Lancet. 2016;387(10027):1513-30.

3. The mysteries of type 2 diabetes in developing countries. Bull World Health Orga. 2016;94(4):241-2.

4. Wan Nazaimoon WM, Md Isa SH, Wan Mohamad WB, Khir AS, Kamaruddin NA, Kamarul IM, et al. Prevalence of diabetes in Malaysia and usefulness of $\mathrm{HbA} 1 \mathrm{c}$ as a diagnostic criterion. Diabet Med. 2013;30(7):825-8.

5. Zhang P, Zhang X, Brown J, et al. Global healthcare expenditure on diabetes for 2010 and 2030. Diabetes Res Clin Pract. 2010;87(3):293-301.

6. Shrivastava SR, Shrivastava PS, Ramasamy J. Role of self-care in management of diabetes mellitus. J Diabetes and Metabolic Dis. 2013;12(1):14

7. Farsaei S, Sabzghabaee AM, Zargarzadeh AH, Amini M. Effect of pharmacist-led patient education on glycemic control of type 2 diabetics: a randomized controlled trial. J Res Med Sci. 2011;16(1):43.

8. Korcegez El, Sancar M, Demirkan K. Effect of a pharmacist-led program on improving outcomes in patients with type 2 diabetes mellitus from Northern Cyprus: A randomized controlled trial. J Manag Care Spec Pharm. 2017;23(5):573-82.

9. Butt M, Mhd Ali A, Bakry MM, Mustafa N. Impact of a pharmacist led diabetes mellitus intervention on $\mathrm{HbA} 1 \mathrm{c}$, medication adherence and quality of life: A randomised controlled study. Saudi Pharm J. 2016;24(1):40-8.

10. Langran T, Nanda N, Bataveljic A, Gonzalez-Durio J. Supporting the management of type 2 diabetes with pharmacist-led reviews: An observational analysis. BMJ Open. 2017;7(3):e013451.

11. Lim PC, Lim K. Evaluation of a pharmacist-managed diabetes medication therapy adherence clinic. Pharm Pract (Granada). 2010;8(4):250-4.

12. Bakar ZA, Fahrni ML, Khan TM. Patient satisfaction and medication adherence assessment amongst patients at the diabetes medication therapy adherence clinic. Diabetes MetabSyndr. 2016;10(2):S139-43.

13. Ministry of Health, Malaysia. Clinical Practice Guidelines: Management of Type II Diabetes Mellitus $5^{\text {th }}$ edition.

14. Ansari A, Thomas S, Goldsmith D. Assessing glycemic control in patients with diabetes and end-stage renal failure. Am J Kidney Dis. 2003;41(3):523-31.

15. Butt M, Ali AM, Bakry MM, Mustafa N. Impact of a pharmacist led diabetes mellitus intervention on $\mathrm{HbA} 1 \mathrm{c}$, medication adherence and quality of life: A randomised controlled study. Saudi Pharmaceutical Journal. 2016;24(1):40-8.

16. Sarkadi A, Rosenqvist U. Experience-based group education in Type 2 diabetes: A randomised controlled trial. Patient education and counseling. 2004;53(3):291-8.

17. Appuhamy JR, Kebreab E, Simon M, Yada R, Milligan LP, France J. Effects of diet and exercise interventions on diabetes risk factors in adults without diabetes: Meta-analyses of controlled trials. Diabetology and metabolic syndrome. 2014;6(1):127.

18. Thent ZC, Das S, Henry LJ. Role of exercise in the management of diabetes mellitus: the global scenario. PloS one. 2013;8(11):e80436.

19. Boulé NG, Haddad E, Kenny GP, Wells GA, Sigal RJ. Effects of exercise on glycemic control and body mass in type 2 diabetes mellitus: A meta-analysis of controlled clinical trials. Jama. 2001;286(10):1218-27.

20. Nelson KM, Reiber G, Boyko EJ. Diet and exercise among adults with type 2 diabetes. Diabetes care. 2002;25(10):1722-8.

21. Thomas N, Alder E, Leese GP. Barriers to physical activity in patients with diabetes. Post Med J. 2004;80(943):287-91. 
Abdullah, et al:: Study about Pharmacist-managed Diabetes clinic

22. Vijan S, Stuart NS, Fitzgerald JT, Ronis DL, Hayward RA, Slater S, et.al. Barriers to following dietary recommendations in Type 2 diabetes. Diabetic Med. 2005;22(1):32-8.

23. Stratton IM, Adler AI, Neil HA, Matthews DR, Manley SE, Cull CA, et al. Association of glycaemia with macrovascular and microvascular complications of type 2 diabetes (UKPDS 35): Prospective observational study BMJ. 2000;321(7258):405-12.

24. American Diabetes Association. Standards of Medical Care in Diabetes-2011. Diabetes Care. 2011;34(1):S11-S61.

Cite this article as: Abdullah MJ, Tew MM, Tan PH, Koh JH, Osman NM, Chan HK. Pharmacist-managed Diabetes Clinic in Malaysia Does the Number of Follow-up Visits Really Matter?. J Pharm Pract Community Med. 2018;4(2):55-9. 